\title{
Nonlinear Analysis of SSR in Wind Power Plants
}

\section{Document Version}

Accepted author manuscript

Link to publication record in Manchester Research Explorer

\section{Citation for published version (APA):}

Chen, Y., Preece, R., \& Barnes, M. (Accepted/In press). Nonlinear Analysis of SSR in Wind Power Plants. In 16th IET International Conference on AC and DC Power Transmission (ACDC 2020)

\section{Published in:}

16th IET International Conference on AC and DC Power Transmission (ACDC 2020)

\section{Citing this paper}

Please note that where the full-text provided on Manchester Research Explorer is the Author Accepted Manuscript or Proof version this may differ from the final Published version. If citing, it is advised that you check and use the publisher's definitive version.

\section{General rights}

Copyright and moral rights for the publications made accessible in the Research Explorer are retained by the authors and/or other copyright owners and it is a condition of accessing publications that users recognise and abide by the legal requirements associated with these rights.

\section{Takedown policy}

If you believe that this document breaches copyright please refer to the University of Manchester's Takedown Procedures [http://man.ac.uk/04Y6Bo] or contact uml.scholarlycommunications@manchester.ac.uk providing relevant details, so we can investigate your claim.

\section{OPEN ACCESS}




\title{
Nonlinear Analysis of SSR in Wind Power Plants
}

\author{
Youhong Chen $^{1^{*}}$, Robin Preece ${ }^{2}$, Mike Barnes $^{3}$ \\ ${ }^{1,2,3}$ Department of Electrical and Electronic Engineering, The University of Manchester, Manchester, M13 9PL, U.K. \\ Email:youhong.chen@postgrad.manchester.ac.uk,robin.preece@manchester.ac.uk,mike.barnes@manchester.ac.uk
}

Keywords: BIFURCATION THEORY, IGE, NYQUIST STABILITY CRITERION, SSR, SMALL-SIGNAL STABILITY

\begin{abstract}
This paper analyzes sub-synchronous resonance (SSR) in future wind power plants (WPPs) using bifurcation theory, a nonlinear analysis approach. SSR, and in particular the Induction Generator Effect (IGE) is likely to occur more frequently as large amounts of wind power are incorporated into power networks, supported by series compensation to improve power transfer capability. A nonlinear $10^{\text {th }}$ order modified IEEE first benchmark model is developed. Bifurcation analysis reveals the critical conditions resulting in IGE are identified as a Hopf bifurcation point, verified by considering the trajectory of system eigenvalues and also through transient time domain simulation. The application of bifurcation theory is compared with the linear Nyquist stability criterion. The results from the Nyquist approach show that both analytical methods are in agreement with each other. However, the nonlinear bifurcation analysis determines system stability with respect to any parameters of interest, whereas the Nyquist stability criterion only determines the system stability at a specific operating point.
\end{abstract}

\section{Introduction}

The large-scale integration of wind power into traditional power networks leads to several challenges. One is the current gap between expected increasing wind power flows and the limited power transfer capacity of existing transmission and distribution networks. Series compensation is the most common and economic means to improve power transfer capacity and is expected to be increasingly used to help integrate large scale wind power plants (WPPs) [1]. However, the introduction of series compensated lines can also result in adverse subsynchronous resonance (SSR). The IEEE divides SSR phenomena into four classifications: 1) self-excitation, due to the induction generator effect (IGE); 2) torsional interaction (TI); 3) subsynchronous control interaction (SSCI); and 4) torsional amplification (TA) [2]. IGE, TI, and SSCI are steady state issues, whereas TA is a transient phenomena. SSCI was initially observed in WPPs in 2008 in Texas and is also called DFIG control participated IGE. It has a similar theoretical basis to conventional IGE-based issues. The work in [3] demonstrates that IGE is the major cause of SSR instability with WPPs.

IGE and SSCI-based issues are typically analysed using eigenvalues, frequency scanning, impedance-based Nyquist analysis, and time domain simulation approaches. Power networks are nonlinear systems, which can be represented as a set of nonlinear algebraic and differential equations. However, two of these commonly used analytical methods (eigenvalue and Nyquist analysis) require linearization which may lead to inaccuracy. The remaining common nonlinear approaches (time domain simulation and frequency scanning) can be computationally cumbersome and require repeated simulations to establish stability boundaries. Power system behaviour can also be investigated using nonlinear bifurcation theory. It can reveal the stability of different stationary points for the system of interest, and describe the cause of the transitions from stable to unstable conditions as different parameters vary.

Local bifurcation theory has been applied to analyse power system low-frequency oscillations and voltage collapse for decades [4]. In addition to voltage collapse, bifurcation theory can be used to examine SSR phenomena in power systems. The SSR phenomena in a single machine infinite busbar (SMIB) system is determined with the application of local bifurcation theory and demonstrated as a Hopf bifurcation in [5]. A Hopf bifurcation is the most commonly encountered bifurcation phenomena, where a complex conjugate eigenvalue pair passes through the imaginary axis. The work in [6] extends bifurcation analysis of SSR phenomena to the IEEE second benchmark model. It further demonstrates that systems may experience severe oscillations due to SSR with increasing series compensation. However, most previous work utilizes bifurcation theory to analyse TI types of SSR issues, for example in [5]-[7], with few studies investigating IGE or SSCI with bifurcation theory, despite their higher probability of occurrence. Also, when modelling the generator, previous research analysed synchronous generators; an induction machine is more suitable for WPP analysis. In addition, there is lack of result-substantiated comparisons between different analysis methodologies.

This paper will investigate IGE in the IEEE first benchmark model using bifurcation theory. Both IGE and SSCI are produced by similar conditions. However IGE is more easily understood and enables comparison of bifurcation theory with other analytical approaches. The conditions causing IGE and TA are also quite similar. Therefore, in order to guarantee that only IGE will occur in the test system, the mechanical representation of the generator will be removed. The results from the bifurcation theory will be verified by eigenvalue analysis and time domain simulation. Furthermore, the results obtained from this nonlinear approach will be compared with results from a linear method - the Nyquist stability criterion - in order to demonstrate the merits and limitations of each approach.

\section{System modelling}

In order to improve the understanding of the different analysis 
techniques presented (in Sections 3 and 4), the system modelling overview is presented first. The study system is adapted from the IEEE first benchmark model [8] and is shown in Fig. 1. Different from the original IEEE first benchmark model, an 892.4 MW type-1 IG based wind farm (instead of a synchronous generator) is connected to the network. The system model can be divided into two subsystems: the induction generator, and the network. The mathematical model of each subsystem is represented as described below. Note that bifurcation theory analysis is based on differential-algebraic equations (DAE), whilst the Nyquist stability criterion is assessed using the transfer function developed from impedance-based modelling.

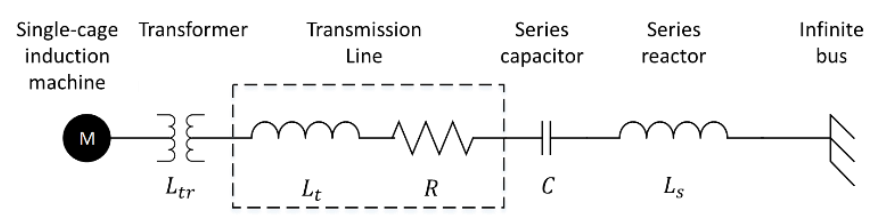

Fig. 1 Modified IEEE first benchmark model

\subsection{State space modelling}

This section will outline the state-space modelling of the study system used for bifurcation theory analysis.

2.1.1 Single-cage IG model: In order to eliminate the occurrence of TI, only the electrical part of the IG is included in the model. The dynamics of a single cage induction generator neglecting flux saturation are given by (1)-(4), with the definitions provided in (5)-(10). [9].

$$
\begin{aligned}
\frac{1}{\omega_{s}} \frac{d}{d t} \lambda_{d s} & =-R_{s} I_{d s}+\lambda_{q s}-V_{d s} \\
\frac{1}{\omega_{s}} \frac{d}{d t} \lambda_{q s} & =-R_{s} I_{q s}-\lambda_{d s}-V_{q s} \\
\frac{1}{\omega_{s}} \frac{d}{d t} \lambda_{d r} & =-R_{r} I_{d r}+\sigma \lambda_{q r} \\
\frac{1}{\omega_{s}} \frac{d}{d t} \lambda_{q r} & =-R_{r} I_{q r}+\sigma \lambda_{d r} \\
\lambda_{d s} & =X_{s} I_{d s}+X_{m} I_{d r} \\
\lambda_{q s} & =X_{s} I_{q s}+X_{m} I_{q r} \\
\lambda_{d r} & =X_{r} I_{d r}+X_{m} I_{d s} \\
\lambda_{q r} & =X_{s} I_{q r}+X_{m} I_{q s} \\
X_{s} & =X_{s \sigma}+X_{m} \\
X_{r} & =X_{r \sigma}+X_{m}
\end{aligned}
$$

In (1)-(10), $\omega_{s}$ is the synchronous rotating speed, $R_{r}$ is the leakage reactance, $X_{s \sigma}$ is the stator leakage reactance, $X_{s}$ is the mutual reactance between the stator and rotor circuit, and $\sigma$ is the slip of the induction generator

Equations (1)-(4) can be arranged into their state space form as (11) to represent the generator sub-system (subscript $G$ ), where $x_{G}=\left[I_{d s}, I_{q s}, I_{d r}, I_{q r}\right]$, and $u_{G}=\left[V_{d s}, V_{q s}\right]$.

$$
\dot{x_{G}}=A_{G} x_{G}+B_{G} u_{G}
$$

2.1.2 AC network: This section develops the state space model of AC network which consists of two subparts: the shunt capacitance, and the rest of the network (including the transformer, transmission line, series reactance and series capacitance),

2.1.2.1 Shunt capacitance: For this study, in order to guarantee that only IGE (and not SSCI) occurs in the system, no voltage control loop is included. Hence, a shunt capacitor is connected to help maintain the generator terminal voltage. Additionally, the shunt capacitor will define the dynamics of the generator terminal voltage and couple the dynamics of the IG and AC network. A shunt capacitor $\left(C_{g}\right)$ connected to the single cage IG terminal bus is represented dynamically in the $d-q$ frame as (12) and (13).

$$
\begin{aligned}
& C_{g} \frac{d}{d t} V_{d s}=I_{d s}-I_{d}+\omega_{s} C_{g} V_{q s} \\
& C_{g} \frac{d}{d t} V_{q s}=I_{q s}-I_{d}+\omega_{s} C_{g} V_{d s}
\end{aligned}
$$

2.1.2.2 Transformer, transmission line, series reactance and series capacitance: The transformer, transmission line and series reactance are modelled as a resistance in series with an equivalent reactance. The dynamic equations representing these network elements are given by (14)-(17), in which $L=$ $L_{t r}+L_{t}+L_{s}$ is the sum of transformer, transmission line and series reactance, $R$ is the transmission line resistance and $C$ is the series capacitance.

$$
\begin{gathered}
L \frac{d}{d t} I_{d}=V_{d s}-R I_{d}+\omega_{s} L I_{q}-V_{c d}-V_{d} \\
L \frac{d}{d t} I_{q}=V_{q s}-R I_{q}+\omega_{s} L I_{d}-V_{c q}-V_{q} \\
C \frac{d}{d t} V_{c d}=I_{d}+\omega_{s} C V_{c q} \\
C \frac{d}{d t} V_{c q}=I_{q}+\omega_{s} C V_{c d}
\end{gathered}
$$

Arranging (14)-(17), the state space form of the network subsystem (subscript $N$ ) is written as (18).

$$
\dot{x_{N}}=A_{N} x_{N}+B_{N} u_{N}
$$

Where $x_{G}=\left[V_{d s}, V_{q s}, I_{d}, I_{q}, V_{c d}, V_{c q}\right], u_{G}=\left[I_{d s}, I_{q s}, V_{b d}, V_{b q}\right]$

2.1.3 Overall state space model: The completed state space model is developed by interfacing two subsystems together. As shown in Fig. 2, the output of the IG-subsystem is the input of the network-subsystem, and vice-versa.

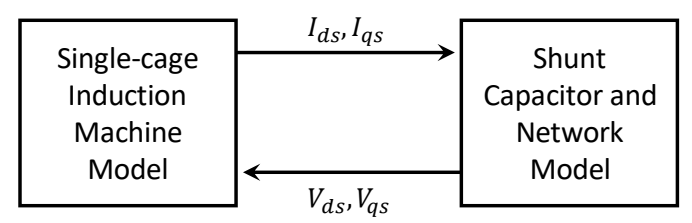

Fig. 2 The structure of completed state space model

The two subsystems can be combined into the overall state space model is written as (19). 


$$
\dot{x}=A x+B u
$$

Where $x=\left[I_{d s}, I_{q s}, I_{d r}, I_{q r}, V_{d s}, V_{q s}, I_{d}, I_{q}, V_{c d}, V_{c q}\right]$

\subsection{Impedance-based modelling}

This section will outline the impedance-based modelling of the study system used for the Nyquist stability criterion.

2.2.1 Single-cage IG model: The per-phase equivalent circuit of the single cage IG can be simplified by neglecting the mutual reactance between the rotor and stator windings since its value is typically much larger than the rotor and stator leakage reactance. Hence, the impedance of a single cage IG seen from the stator circuit can be expressed as (20) in the Laplace domain.

$$
Z_{G}(s)=\frac{R_{r}}{\sigma(s)}+R_{s}+\left(L_{s \sigma}+L_{r \sigma}\right) s
$$

The slip is dependent on the rotor rotating speed $\omega_{r}$ and stator synchronous frequency $\omega_{s}$. However, the rotor speed is considered constant in this study as only the electrical circuit is analysed and the mechanical system dynamics are much slower, resulting in (21).

$$
\sigma(s)=\frac{s-j \omega_{r}}{s}
$$

2.2.2 AC Network: The equivalent circuit of the network consists of a resistance $R$, capacitor $C$ and inductance $L$ connected in series. The inductance is the sum of transformer, transmission line and series inductance. In this approach, the integration of the AC network and IG is not needed and hence the shunt capacitor is not needed. Hence, the per-phase impedance of the network can be expressed in the Laplace domain as (22).

$$
Z_{N}(s)=R+L s+\frac{1}{C s}
$$

2.2.3 Overall impedance model: When analysing the IGE with the Nyquist stability criterion, $Z_{N}(s)$ is the source impedance, while $Z_{G}(s)$ is the load impedance since the network is the source of the SSR. Therefore, the transfer function $Z_{N}(s) /$ $Z_{G}(s)$ is used.

\section{Bifurcation analysis of IGE}

In any dynamic system, the system characteristics will qualitatively change from the original state to another state as some of the system parameters vary. For example, in power systems, with the load demand increasing, the voltage will gradually decrease until finally voltage collapse occurs. The point right before the system exhibits qualitative change is considered to be a critical stage which can be identified using bifurcation theory. This paper focuses on investigating IGE issues, so only local Hopf bifurcation theory is considered.

A power system is a complex system with many nonlinear components resulting in many nonlinear dynamic phenomena. Considering the test system developed in Section 2, the dynamic model of a power system is represented by a set of nonlinear differential equations as in (23), where $x$ is the vector of system state variables and $\mu$ is a scalar control parameter [10].

$$
\dot{x}=F(x, \mu)
$$

With $\mu$ varying, the equilibrium point and the Jacobian matrix $\left(\frac{d F}{d x}\right)$ changes as a function of $\mu$. At each equilibrium point, the derivative of all system state variables is zero, indicating system stability with constant system parameters. The system remains stable if the real part of all the eigenvalues of the Jacobian matrix are negative at this equilibrium point. The system may reach a critical point when a pair of eigenvalues in a Jacobian matrix system has pure imaginary conjugate roots, where the system starts to experience small magnitude oscillation as given in (24) with $I_{n, m}$ given in (25).

$$
\begin{gathered}
\operatorname{det}\left\{j w I_{n, m}-J(x, \mu)\right\}=0 \\
I_{n, m}=\left[\begin{array}{cc}
I_{n} & 0_{n \times m} \\
0_{m \times n} & 0_{m}
\end{array}\right]
\end{gathered}
$$

When a pair of complex conjugate eigenvalues crosses the imaginary axis into the right-half plane, the system will start to lose stability. This phenomenon is classified as a Hopf bifurcation and the conditions for determining it are that:

1. $F\left(x_{c}, \mu_{c}\right)=0$.

2. A pair of complex eigenvalues in the Jacobian matrix has pure imaginary conjugate roots, i.e. eig $\left(\frac{d F}{d x_{c}}\right)= \pm j \omega$.

3. $d\left(\operatorname{Re}\left(e i g\left(\frac{d F}{d x_{c}}\right)\right)\right) \neq 0$.

When these three conditions are satisfied, it is guaranteed that the system has a pair of complex eigenvalues of the Jacobian matrix crossing the imaginary axis. That is to say, a Hopf bifurcation will result in a qualitative change in system stability [4]. Hence, $\mu_{c}$ is determined as the Hopf bifurcation point value. The sign of a third derivative equation determines the crossing direction of the eigenvalues. If positive, the complex conjugate eigenvalues pass the imaginary axis towards right half plane, indicating the system will become unstable with a start of limit cycles. When it is negative, the complex conjugate eigenvalues move to the left half plane and the system changes from unstable to stable with an end to limit cycle behaviour.

\section{Impedance-based Nyquist stability criterion analysis of IGE}

To apply impedance-based stability analysis, the system must be divided into two subsystems: the source and the load. The source consists of an ideal voltage source $(V)$ in series with an impedance $\left(Z_{s}\right)$, while the load is modelled as a series connected impedance $\left(Z_{l}\right)$. The current flowing in the system can be expressed as (26) [11]

$$
I(s)=\frac{V(s)}{Z_{s}(s)+Z_{l}(s)}=\left(\frac{V(s)}{Z_{l}(s)}\right)\left(\frac{1}{1+Z_{s}(s) / Z_{l}(s)}\right)
$$

System stability is dependent on the second term of the current transfer function as in (27) with $\frac{V(s)}{Z_{l}(s)}$ assumed to be stable. 


$$
H(s)=\frac{1}{1+Z_{s}(s) / Z_{l}(s)}
$$

Therefore, the system is stable if $Z_{s}(s) / Z_{l}(s)$ satisfies the Nyquist stability criterion. That is to say, either the Nyquist map of $Z_{s}(s) / Z_{l}(s)$ does not encircle the point $(-1, j 0)$ or the number of counter-clockwise encirclement of $(-1, j 0)$ must be equal to the number of unstable poles of $Z_{s}(s) / Z_{l}(s)$.

Generally speaking, if the Nyquist map $Z_{s}(s) / Z_{l}(s)$ encircles the point $(-1, j 0)$ once, the system is unstable. Assuming instability, there is a frequency $\omega_{0}$ which makes $Z_{s}\left(j \omega_{0}\right) /$ $Z_{l}\left(j \omega_{0}\right)$ purely real and smaller than -1 . So (27) becomes (28), which can be formulated as (29) [12].

$$
\begin{gathered}
\frac{Z_{s}\left(j \omega_{0}\right)=R_{s}}{Z_{l}\left(j \omega_{0}\right)=R_{l}}<-1 \\
R_{s}+R_{l}<0
\end{gathered}
$$

Therefore, according to the impedance-based Nyquist stability criterion, the system is unstable when the sum of the system source and load apparent impedance is negative. Applying this to the impedance model developed in Section 2.2, the Nyquist stability criterion for IGE becomes (30).

$$
\frac{R_{r}}{s \sigma(s)}+R_{s}+R<0
$$

This is consistent with the explanation of IGE from the network operator view. This states that IGE occurs when the rotor resistance seen from the stator winding terminal is negative, and is larger than the net impedance of the system resistance and stator resistance - as is shown in equation (30) [13].

\section{Case study}

The study system analysed in this paper is adapted from the IEEE first benchmark model as previously described.

For the bifurcation analysis, the complete nonlinear dynamic model described in Section 2.1 was formed in MATLABR2018a. The bifurcation analysis is completed using the software package MATCONT [14]. The series connected capacitance $X_{c}$ and the resistance in transmission line $R$ were considered as bifurcation parameters. The series capacitance varied from 14-100\% of the reactance in the transmission line, while transmission line resistance changes from $0-1$ p.u.

For the verfiying time domain simulation, the modified IEEE first benchmark model is simulated in DIgSILENT PowerFactory 2018. A $0.075 \mathrm{~s}$ self-clearing three-phase fault at the generator terminal bus is used as an initiating disturbance. The FFT is carried out based on time domain simulation results and hence is also carried out in DIgSILENT PowerFactory 2018.

The application and analysis is presented in three stages. Firstly, one-parameter bifurcation analysis (with series capacitance selected as the bifurcation parameter) is performed. These results are verified considering the trajectory of eigenvalues of the Jacobian matrix and time domain simulations. Secondly, two-parameter bifurcation analysis is performed with both series capacitance and transmission line resistance considered as bifurcation parameters. Finally, the results from the linear Nyquist stability criterion approach are compared with those obtained using nonlinear bifurcation analysis.

\subsection{Bifurcation analysis}

5.1.1 One-parameter analysis: In this first case, the series capacitance $X_{c}$ is chosen as the bifurcation parameter. The value of $X_{c}$ varies from $14 \%$ to $100 \%$ of the reactance in the transmission line, i.e. from 0.1 p.u. to 0.7 p.u. All other system parameters remain constant. Fig. 3 shows the change in generator terminal q-axis voltage as $X_{c}$ varies. The solid line in Fig. 3 indicates the system is stable, while the dashed line indicates the system is unstable. There is a Hopf bifurcation point when series capacitance is 0.5507 p.u., marked on Fig. 3 as HB. This type of Hopf bifurcation point, as well as the transverse direction of the movement of the complex conjugate eigenvalues, can be checked by calculating the first Lyapunov exponent [4].The sign of the exponent is positive in this case and so it is a subcritical Hopf bifurcation point - meaning that the complex conjugate eigenvalues pass through the imaginary axis from left to right. This further indicates that the system becomes unstable at this Hopf bifurcation point as $X_{c}$ increases. Physically this means that the system will show IGE phenomena when $X_{c}>0.5507$ p.u.

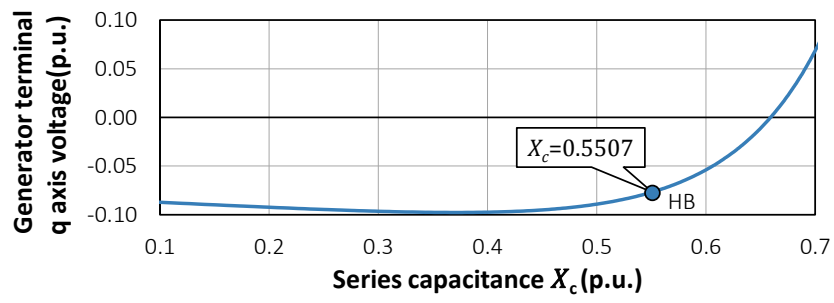

Fig. 3 One-parameter bifurcation analysis with $X_{c}$ as the bifurcation parameter. The solid line represents stable fixed points, whilst the dashed line indicates unstable fixed points.

To support this result, the trajectory of a pair of conjugate eigenvalues in the Jacobian matrix as a function of series capacitance is shown in Fig. 4, and the pair of essential complex conjugate eigenvalues are also displayed. The pair of complex conjugate eigenvalues continue to move closer to the imaginary axis as the reactive power compensation level increases and consequently passes to the right half plane at the Hopf bifurcation point. This indicates a limit cycle begins at this bifurcation point and the original stable system becomes unstable, in agreement with the earlier bifurcation analysis.

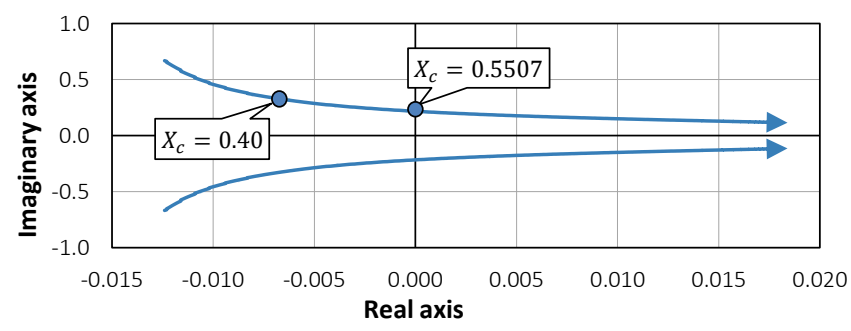

Fig. 4 The trajectory of complex conjugate eigenvalue pair with increasing $X_{c}$

In order to further verify the bifurcation analysis results, time domain simulations are performed. The simulation results 
when $X_{c}=0.4$ p.u and $X_{c}=0.6$ p.u are shown in Fig. 5. Fig. 5(a) shows that when $X_{c}=0.4$ p.u., lower than the HB point, the generator terminal voltage regains stability following the disturbance (the self-clearing fault at time $t=1 \mathrm{~s}$ ). However, when $X_{c}=0.6$ p.u., which is above the HB point critical value, as shown in Fig. 5(b), the post-disturbance voltage displays oscillations with increasing magnitude.

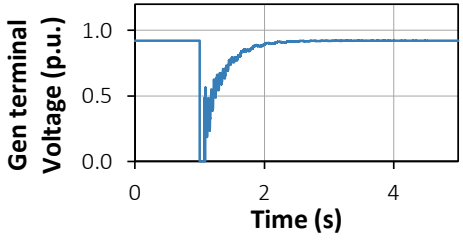

(a)

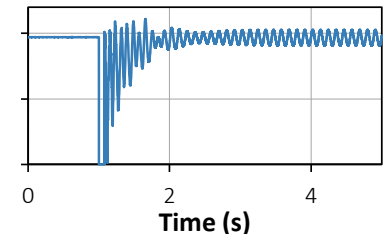

(b)
Fig. 5: Generator terminal voltage with (a) $X_{c}=0.4 p . u$. and (b) $X_{c}=0.6 p . u$.

Fast Fourier Transform (FFT) analysis of the post-disturbance generator terminal phase A voltage is shown in Fig. 6. The results show a spike at $51 \mathrm{~Hz}$ with a value of $0.01795 \mathrm{p}$.u. This is because when IGE occurs, the apparent negative rotor resistance will amplify the resonant frequency component (in this case $51 \mathrm{~Hz}$ ). Additionally, the resonant frequency interacts with the nominal frequency $(60 \mathrm{~Hz})$ resulting in a third supersynchronous oscillation in the network. This is the cause of the spike at $69 \mathrm{~Hz}$ with a value of 0.04073 p.u. in Fig. 6. The results shown in these different time domain analysis are coherent with the bifurcation analysis result.



Fig. 6 FFT of generator terminal phase A voltage with $X_{c}=$ 0.6 p.u. Note that the $y$-axis is scaled such that the FFT at $60 \mathrm{~Hz}$ is off-scale. Frequencies outside 40-80 Hz are not shown.

5.1.2 Two-parameter analysis: For this second study case, the transmission line resistance $R$ is also considered as a bifurcation parameter. The value of $R$ is considered in the range from 0 to 1 p.u. The Hopf bifurcation point from Fig. 3 (and Section 5.1.1) is used as an initial point to track the trajectory of the HB point for variations in both $X_{c}$ and $R$. This HB trajectory is shown as Fig. 7.

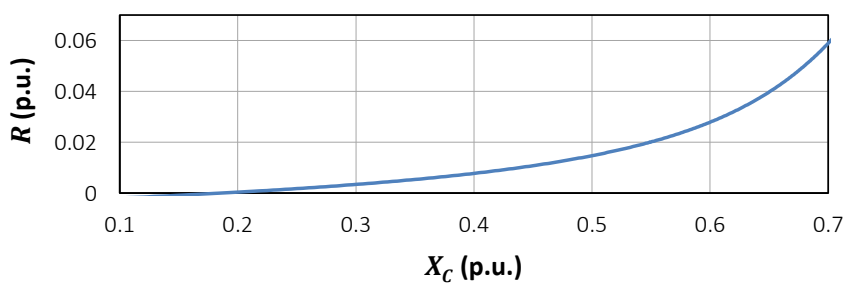

Fig. 7 Two-parameter $\left(X_{c}\right.$ and $R$ ) bifurcation analysis.

Every point located on the line in Fig. 7 is a subcritical Hopf bifurcation point. Hence, compared with the one-parameter bifurcation analysis case, an IGE boundary instead of an IGE critical point is identified. The area above the HB trajectory line in Fig. 7 (and contained within the feasible range of the bifurcation parameters $X_{c}$ and $R$ ) represents the stable zone in which IGE will not occur. As can be seen from Fig. 7, as $X_{c}$ increases, the trajectory of the HB point moves to greater values of $R$. These analysis results agree with the view from the electrical network operator: that with large resistance in the network or lower series reactive power compensation levels, IGE is less likely to occur [3].

To help confirm the two-parameter bifurcation analysis results, time domain simulations are performed for two points:

1. Within the stable region: $X_{c}=0.2$ p.u., $R=0.2$ p.u.

2. Within the unstable region: $X_{c}=0.6$ p.u., $R=0.01$ p.u.

Fig. 8 (a) displays the results for the first (stable region) case. All oscillations in the post-disturbance voltage are very quickly damped and the system reaches steady state again. Thus, there is no IGE occurring in the system under these conditions. The results for the second (unstable region) case are shown in Fig. 8(b). It can be seen that the generator terminal voltage experiences significant oscillations after the disturbance and the system becomes unstable, suggesting the occurrence of IGE in the system. These results from the time domain simulations are consistent with the bifurcation analysis.



(a)

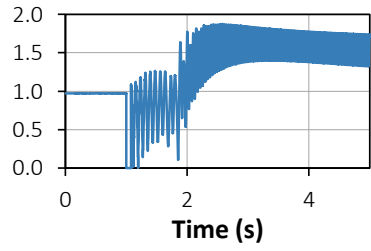

(b)
Fig. 8 Generator terminal voltage with (a) $R=0.01$ p.u, $X_{c}=$ 0.6 p.u. and (b) $R=0.2$ p.u, $X_{c}=0.2$ p.u.

\subsection{Comparison with Nyquist analysis}

Finally, the Nyquist stability criterion is applied to the same study system with exactly the same parameters to enable a direct comparison. For IGE phenomena, network is the resonant frequency source and the IG is the network that boosts it. Hence, based on the theory developed from Section $4, Z_{s}(\mathrm{~s})=$ $Z_{N}(s)$ and $Z_{l}(\mathrm{~s})=Z_{G}(s)$. The transfer function of $Z_{N}(s) /$ $Z_{G}(s)$ is derived and the Nyquist plots for different parameter cases are shown in Fig. 9. In this case, only $X_{C}$ is considered as the scaling parameter, with $R=0.02$ p.u. In order to compare the results from the bifurcation analysis, the Nyquist stability criterion is applied to the system when the series capacitances are 0.4 p.u and 0.6 p.u. respectively, as in Section 5.1.1.

It is shown in Fig. 9(a) that, when $X_{C}=0.4$ p.u, the Nyquist mapping of $Z_{N}(s) / Z_{G}(s)$ does not encircle $(-1, \mathrm{j} 0)$. This suggests the system does not have IGE issues and will remain stable. When $X_{C}$ is increased to 0.6 p.u., the Nyquist plot in Fig. 9 (b) encircles the point $(-1, j 0)$ once, which implies the system will suffer from IGE issues. These results confirm the conclusions from the bifurcation analysis and transient simulation. 


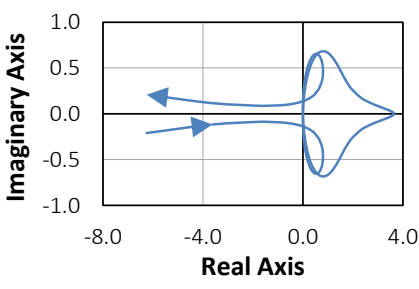

(a)

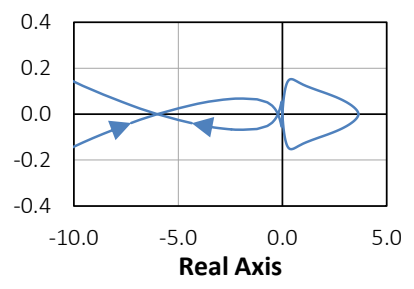

(b)
Fig. 9 Nyquist plot of $Z_{N}(s) / Z_{G}(s)$ with (a) $X_{c}=0.4 p$.u. and (b) $X_{c}=0.6 p . u$.

Both results from the bifurcation analysis and the Nyquist stability criterion determined the system stability correctly. However, the bifurcation analysis shows several unique advantages over the Nyquist stability criterion. Firstly, the bifurcation analysis is a graphic and 'dynamic' analytical approach. With one simulation, system stability with respect to one parameter varying, or even multiple parameters varying, is obtained. The critical point for system stability (the Hopf bifurcation point in a one-parameter analysis or critical boundary in a two-parameter analysis) is also identified within one simulation. However, compared to the bifurcation analysis, the Nyquist stability criterion works more like a graphic 'static' analytical method. This way, in a single simulation, system stability under a single parameter setting is determined. If information about system stability as parameters vary is desired then multiple simulations are required with additional regulation of parameter settings.

In addition, the Nyquist stability criterion requires a linear transfer function, while bifurcation analysis can be carried out on both nonlinear and linear differential equations. However, in a power system, numerous power devices are nonlinear and hence the accurate modelling of those components is also nonlinear. Although both analytical approaches showed the same results when determining system stability, the linearization of the nonlinear power system may result in errors in the results.

\section{Conclusion}

In this paper, the bifurcation theory is applied to analyse the IGE type of SSR issues in the modified IEEE first benchmark model. The completed $10^{\text {th }}$ order model of differential equations in state space form is developed for the bifurcation analysis. When only series capacitance is considered as the bifurcation parameter, a Hopf bifurcation is identified at series capacitance $X_{c}=0.5507$ p.u. The time domain simulation verifies the bifurcation analysis results. This also demonstrates that the Hopf bifurcation point in this case stands for the IGE point. In the two-parameter analysis, the Hopf bifurcation trajectory (or IGE boundary) is found when both the series capacitance and resistance of the transmission line are selected as bifurcation parameters. Moreover, the bifurcation analysis has been evaluated against the results from the Nyquist stability criterion. The results indicate a strong agreement from both of the analytical approaches. However, a comparison of the two procedures suggests that the bifurcation analysis has the advantage of providing more information regarding system stability.

\section{Acknowledgements}

Youhong Chen acknowledges the financial support from EPSRC(EP/L016141/1) through the Power Networks Centre for Doctoral Training.

\section{References}

[1] N. Bacalao, A. W. Galli, M. Hutson, and R. Nadira, "Considerations on the use of HVDC for CREZ transmission," in 2009 IEEE/PES Power Systems Conference and Exposition, 2009, pp. 1-7.

[2] "Reader's guide to subsynchronous resonance," IEEE Trans. Power Syst., vol. 7, no. 1, pp. 150-157, 1992.

[3] L. Fan, R. Kavasseri, Z. L. Miao, and C. Zhu, "Modeling of DFIG-Based Wind Farms for SSR Analysis," IEEE Trans. Power Deliv., vol. 25, no. 4, pp. 2073-2082, Oct. 2010.

[4] H. G. Kwatny, R. F. Fischl, and C. O. Nwankpa, "Local Bifurcation in Power Systems: Theory, Computation, and Application," Proc. IEEE, vol. 83, no. 11 , pp. 1456-1483, 1995.

[5] W. Zhu and W. A. Mittelstadt, "Hopf bifurcations in a SMffi power system with SSR," IEEE Trans. Power Syst., vol. 11, no. 3, pp. 1579-1584, 1996.

[6] A. M. Harb and M. S. Widyan, "Modern nonlinear theory as applied to SSR of the IEEE second benchmark model," in 2003 IEEE Bologna PowerTech - Conference Proceedings, 2003, vol. 3, pp. 967-973.

[7] R. C. Mala, N. Prabhu, and H. V. Gururaja Rao, "Hopf bifurcations of Subsynchronous resonance in a hybrid series compensated system with SSSC-ES," in Proceedings of IEEE International Conference on Technological Advancements in Power and Energy, TAP Energy 2015, 2015, pp. 65-72.

[8] P. M. Anderson, B. L. Agrawal, and J. E. Van Ness, Subsynchronous resonance in power systems. IEEE Press, 1990.

[9] A. K. Moharana, "Subsynchronous resonance in wind farms," The University of Western Ontario, 2012.

[10] V. Ajjarapu and B. Lee, "Bifurcation theory and its application to nonlinear dynamical phenomena in an electrical power system," in [Proceedings] Conference Papers 1991 Power Industry Computer Application Conference, pp. 312-319.

[11] J. Sun, "Impedance-based stability criterion for gridconnected inverters," IEEE Trans. Power Electron., vol. 26, no. 11, pp. 3075-3078, 2011.

[12] L. Fan and Z. Miao, "Nyquist-stability-criterion-based SSR explanation for type-3 wind generators," IEEE Trans. Energy Convers., vol. 27, no. 3, pp. 807-809, 2012.

[13] K. R. Padiyar, Analysis of Subsynchronous Resonance in Power Systems, 1st ed. Boston, MA: Springer US, 1999.

[14] "MatCont download | SourceForge.net." [Online]. Available: https://sourceforge.net/projects/matcont/. [Accessed: 02-Nov-2019]. 